\title{
A Comparison of Nutritional Status of Women Suffering from Mental IIIness in Urban and Rural Areas of Bangladesh
}

\author{
Khan Redzwan Habib \\ World Health Organisation, Dhaka, Bangladesh
}

Email address:

khan.redz@gmail.com, habibk@who.int

To cite this article:

Khan Redzwan Habib. A Comparison of Nutritional Status of Women Suffering from Mental Illness in Urban and Rural Areas of Bangladesh. International Journal of Nutrition and Food Sciences. Vol. 6, No. 2, 2017, pp. 65-70. doi: 10.11648/j.ijnfs.20170602.12

Received: January 8, 2017; Accepted: January 17, 2017; Published: February 20, 2017

\begin{abstract}
About $16.05 \%$ of the adult population in Bangladesh suffers from mental illness. $74 \%$ of these mentally impaired patients live in rural areas and are socially disadvantaged. Studies have identified mental disorder to be a risk factor for involuntary weight loss and malnutrition. This study aims to explore the differences in nutritional status among women suffering from mental illness from urban and rural areas based on their socio-economic status. This cross sectional study has been conducted for over a period of four months that covered six divisions of the country. A total of 73 participants aged from 15-60 were purposively selected to represent urban \& well off population and the rest 73 were selected to represent rural \& socially disadvantaged population. Height, weight and BMI were significantly lower in all participants from rural settings. About $18 \%$ of all participants from rural areas have a BMI $18 \mathrm{~kg} / \mathrm{m}^{2}$ or less. Mean body fat percentage (BF\%) is significantly higher in all subgroups from urban areas $(\mathrm{P}<0.001)$ comparing to those from rural settings. No significance was observed in EI:BMR ratios. Mean EI:BMR ratio was over 1.4 in all subgroups from both urban and rural settings except for 46-60 years participants from urban areas. The highest EI:BMR ratio has been observed in the subgroup of 31-45 years aged women from urban areas. The study has identified that mentally impaired women even from well off families or from urban settings have an inferior nutritional status comparing to those of healthy women from same settings. The nutritional status of mentally impaired women from rural areas or from lower socio-demographic settings is even worse. Low BMI and low energy intake indicates vulnerability of mentally impaired women to malnutrition in Bangladesh.
\end{abstract}

Keywords: Mental Disorder, Malnutrition, Socio-Economic Group, Anthropometric Analysis, Health Inequality

\section{Introduction}

Bangladesh is world's eighth most populated country and fifth most populated country in Asia. Total population of the country as of 2016 is $162,903,941$ [1]. The country consists of Eight divisions and sixty-four districts [2]. Despite progress made in this sector malnutrition rates in Bangladesh are among the highest in the world. Malnutrition among women is also extremely prevalent. Over $50 \%$ women in the country suffer from chronic energy deficiency [3]. Negative energy intake and chronic energy malnutrition are even higher among women low socio-economic group specially from rural areas $[4,5]$. Studies suggest that this situation has seen little improvement in past 20 years.

Numerous studies have suggested a strong correlation between mental impairment and malnutrition. These studies have identified mental disorder to be a risk factor for involuntary weight loss [6-8]. A study conducted in Sweden in 2009 found mental disorders to predict malnutrition in community [9], whereas a German study conducted in the same year found no significant difference in mean malnutrition score between people with or without mental disorders [10].

Prevalence of mental illness varies from $6.5 \%$ to $31 \%$ among adults in Bangladesh [11]. According to national mental health survey conducted in 2003-2005, about $16.05 \%$ of the adult population has been suffering from mental illness. Following the survey mental health policy, strategy and plan 
have been approved in Bangladesh in the year 2006. 39\% of the population is under the age of 15 and $6 \%$ is over 60 years. Alarmingly $74 \%$ of these mentally impaired patients live in rural areas and are socially disadvantaged. The majority of the patients seeking medical help are psychotic. The neurotic patients most of the times do not seek medical help due to social stigma and therefore remains undiagnosed and suffer from various physical health problems including malnutrition [12]. Both urban and rural groups possess a higher vulnerability of mental illness among women compared to men [13]. Prevalence of mental illness among women are even higher among economically disadvantaged group and in rural areas [11, 14]. Several studies have been identified comparing nutritional status of women from urban and rural areas in Bangladesh [15, 16]. But little published data has been identified in literature review comparing malnutrition status of women suffering from mental illness in urban and rural areas in Bangladesh. This study therefore, aims to explore the differences in nutritional status among women suffering from mental illness from urban and rural areas based on their socio-economic status. The findings of the study are believed to develop a better idea about malnutrition status among mentally impaired women in Bangladesh. This will eventually lead to develop better policy to understand needs of this disadvantaged group both at the country level and internationally.

\section{Method}

The study was conducted over a period of four months, starting from 18 February 2015 to 21 May 2015. This is a cross sectional study that covered six divisions out of eight of the country. The rest of the two divisions could not be covered due to shortage of time and finance. Therefore, the study areas were selected purposively. Three divisional headquarters were selected to represent the urban population and three upazillas from other three divisions were selected to represent the rural study population. Ethical Approval was taken from Ministry of Health and Family Welfare and from corresponding Civil Surgeons of selected districts.

A total of 146 mentally impaired women aged from 15-60 were selected. These women have been identified through psychiatrists working in these selected towns and other NGOs working with mentally impaired patients. Among the study participants 73 were from urban areas who were purposively selected to represent the well off group of the country. These patients belong to households in which family members either hold a government or non-government job or own a business. All of these participants lives in buildings equipped with modern facilities. The rest 73 were also purposively selected and were from three upazillas who represent the disadvantaged group of the society. These participants belong to households where family members are day-labourers or housemaids or possess small business.

At first stage of the study a questionnaire was developed to address the economic status, status of mental impairment, additional health issues and age of onset of mental illness and level education if any. Due to their mental impairment informed written consent was taken either from parents or in absence of parents from other legal guardians of all study participants. Information on the participant's energy intake has been collected using local family planning assistants (FWAs). The FWAs were first given idea on questionnaire and were later taught on data collection procedure, specially on how to take height and weight measurements. Later they have collected data during their visits to the participants' houses.

The FWAs collected data on dietary habits and daily energy intake on three separate house visits. The participants were informed on the day of house visit to minimize bias as they might have a tendency to alter diet if they would knew about house visits earlier. Height and weight measurements were taken on the first and the last visits. Height and weight were used to calculate Body Mass Index (BMI) as recommended by World Health Organisation (WHO) and Centres for Disease Control and Prevention (CDC). The equation to calculate BMI:

$B M I=$ weight $(\mathrm{kg}) /[\text { height }(\mathrm{m})]^{2}$ (Formula: 1$)$

BMI values $<18.5 \mathrm{~kg} / \mathrm{m}^{2}$ was considered underweight and BMI values $\geq 25 \mathrm{kh} / \mathrm{m}^{2}$ was considered as over weight [17].

Basal Metabolic Rate (BMR) was calculated using HarrisBenedict equation which was revised by Mifflin and St. Jeor in 1990 [18]. This equation has been used since it has been proven to be more accurate than other existing formulas and also considering the fact that taking skin fold thickness for calculating BMR will be difficult from mentally impaired patients as they might not be willing to go through the physical examination.

Mifflin and St. Jeor equation for calculating BMR in women:

$B M R=10 \mathrm{x}$ weight $(\mathrm{kg})+6.25 \mathrm{x}$ height $(\mathrm{cm})-5 \mathrm{x}$ age (years) - 161 (Formula: 2) [18]

For calculating Body Fat Percentage $(\mathrm{BF} \%)$ the following equations has been used for the same reasons mentioned above.

Adult $B F \%=(1.20 \times \mathrm{BMI})+(0.23 \times$ Age $)-(10.8 \times$ sex $)$ -5.4 (Formula: 3 )

[where for women, sex $=0]$ [19]

\section{Result}

Mean monthly family income of the family of the study participants was: in case of urban 469 USD and in case of rural area it was 36 USD (at present 1 USD $=79$ BDT approximately). The mean monthly expenditure for food in urban areas was 110 USD and in rural areas it was 29 USD. Among the study participants majority fell into 31-45 years barrier. The lowest number of participants was from 46-60 years of age. The age distribution of the study participants has been shown in figure 1 . 


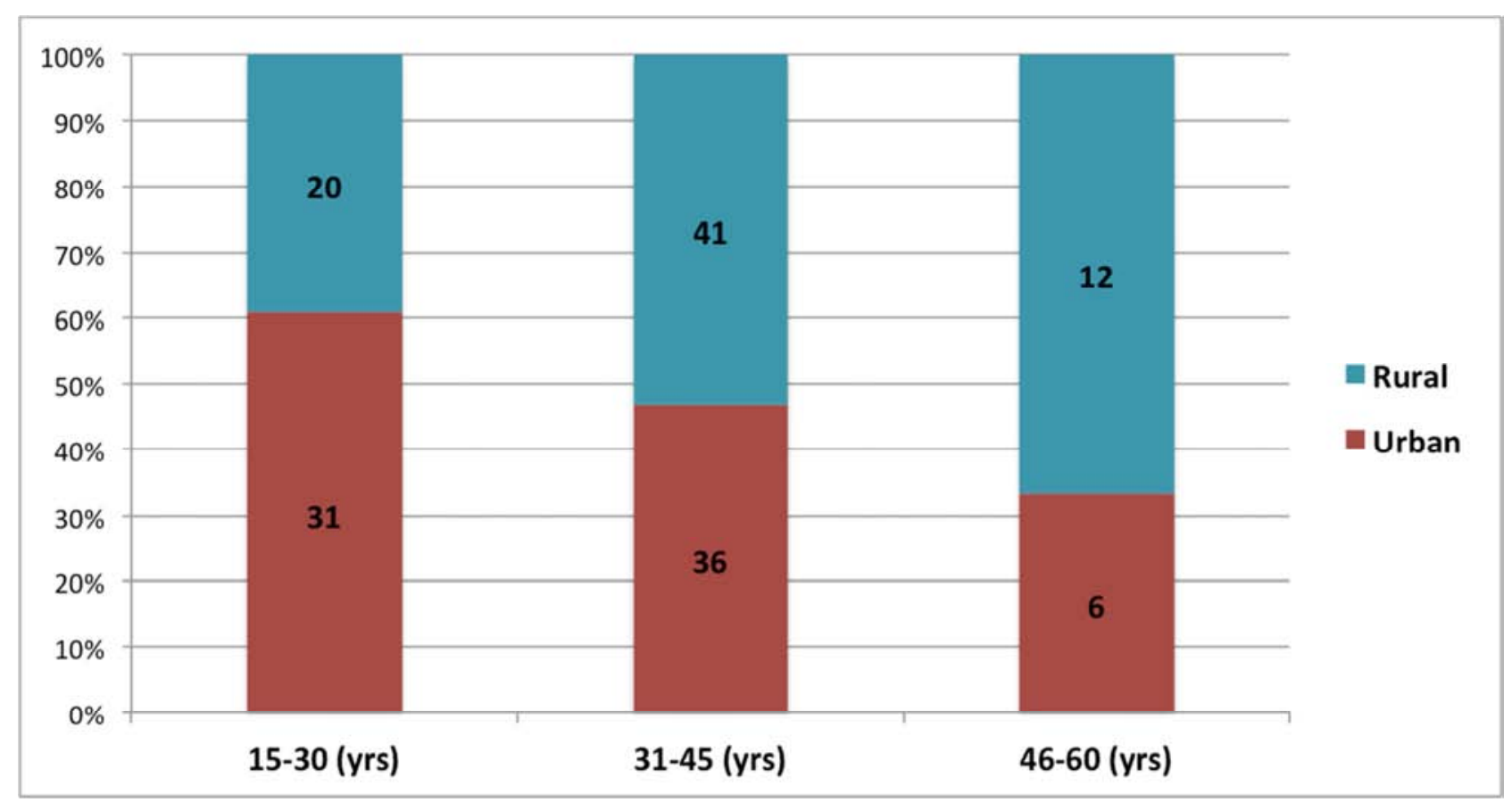

Figure 1. Age distribution of the study participants.

The age of onset of mental impairment is found to be significantly higher from 15 to 45 years in both urban and rural settings. In later years of life onset of mental disorder found in this study is significantly low in both settings. The age of onset of mental impairment has been categorized into three subgroups which has been shown in table 1:

Table 1. Age of onset of mental impairment and percentage of total participants.

\begin{tabular}{llll}
\hline & $\mathbf{1 5 - 3 0}$ (years) & $\mathbf{3 1 - 4 5}$ (years) & $\mathbf{4 6 - 6 0}$ (years) \\
\hline Urban & $34(46.57 \%)$ & $39(53.42 \%)$ & $0(0 \%)$ \\
Rural & $32(43.83 \%)$ & $36(49.31 \%)$ & $5(6.84 \%)$ \\
\hline
\end{tabular}

Out of 73 participants from rural settings 39 (53.4\%) are either currently married or have been married before. For urban settings this number is 19 out of 73 (26\%). Among all participants who have got married 33 out of 39 (84.61\%) from rural areas and 12 out of $19(63.15 \%)$ participants from urban areas have children (table 2).

Table 2. Marital and Pregnancy status,

\begin{tabular}{|c|c|c|c|c|c|c|c|}
\hline & \multicolumn{4}{|c|}{ Marital status } & \multicolumn{3}{|c|}{ Pregnancy status } \\
\hline & Unmarried & Married & Divorced & Widowed & None & Single & Multiple \\
\hline Urban & 54 & 11 & 7 & 1 & 61 & 9 & 3 \\
\hline Rural & 34 & 14 & 17 & 8 & 40 & 16 & 17 \\
\hline
\end{tabular}

This study has identified several associated diseases in women from both urban and rural settings (table 3 ). In both settings a major portion of women with mental illness are suffering from diseases arising from lack of cleanliness or self-hygiene. About 24\% women from urban areas and $37 \%$ women from rural areas are suffering from skin diseases like Psoriasis, Seborrheic Eczema, Scabies and fungal infections. Prevalence of other chronic diseases is common among these patients. $37 \%$ women with mental impairment in urban areas have multiple chronic conditions and for rural areas the number is even higher (44\%). These chronic conditions include cardiovascular diseases, diabetes, asthma, chronic urinary tract infection etc. Alarmingly for $22 \%$ women suffering from mental illness in rural areas have undiagnosed underlying chronic diseases.

Table 3. Associated diseases.

\begin{tabular}{|c|c|c|c|c|c|c|}
\hline & Skin diseases & Cardiovascular diseases & Lung diseases & Diabetes & Multiple chronic conditions & Unknown \\
\hline Urban & $17(23.2 \%)$ & $23(31.5 \%)$ & $6(8.21 \%)$ & $13(17.8 \%)$ & $27(36.9 \%)$ & $7(9.5 \%)$ \\
\hline Rural & $27(36.9 \%)$ & $19(26 \%)$ & $18(24.6 \%)$ & $7(9.5 \%)$ & $32(43.8 \%)$ & $22(30.1 \%)$ \\
\hline
\end{tabular}

The study has identified significant differences $(\mathrm{P}<0.005)$ in dietary habit of the study participants from urban and rural areas. Participants from urban areas have higher intake of all food items. Only for cereals and other carbohydrates intake 
two groups show no significant difference $(\mathrm{P}>0.005)$. But for all other food items intake urban participants have a much higher daily energy intake. Meat, eggs, milk and fruits are absent from daily dietary intake of the study participants from rural settings (table 3 ).

Table 4. Daily food consumption (gm/day).

\begin{tabular}{lllllllll}
\hline & Cereals & Other Carbohydrates & Meat & Fish & Eggs & Milk & Fruits & Vegetables \\
\hline Urban & 334 & 49.2 & 55.3 & 40.7 & 22.8 & 43.7 & 79.4 & 60.2 \\
Rural & 319.2 & 36.6 & 0 & 13.9 & 0 & 0 & 0 & 30.6 \\
\hline
\end{tabular}

Height, weight and BMI were significantly lower in all participants from rural settings (figure 2). About $18 \%$ of all participants from rural areas have a BMI $18 \mathrm{~kg} / \mathrm{m}^{2}$ or less which means these participants are under weight. It is to be noted that none of the study participants from urban settings has a BMI below $18 \mathrm{~kg} / \mathrm{m}^{2}$. On the other hand significant number of participants from the urban areas are over weight (28.77\%). Lower BMI is more prevalent among study participants from rural areas.

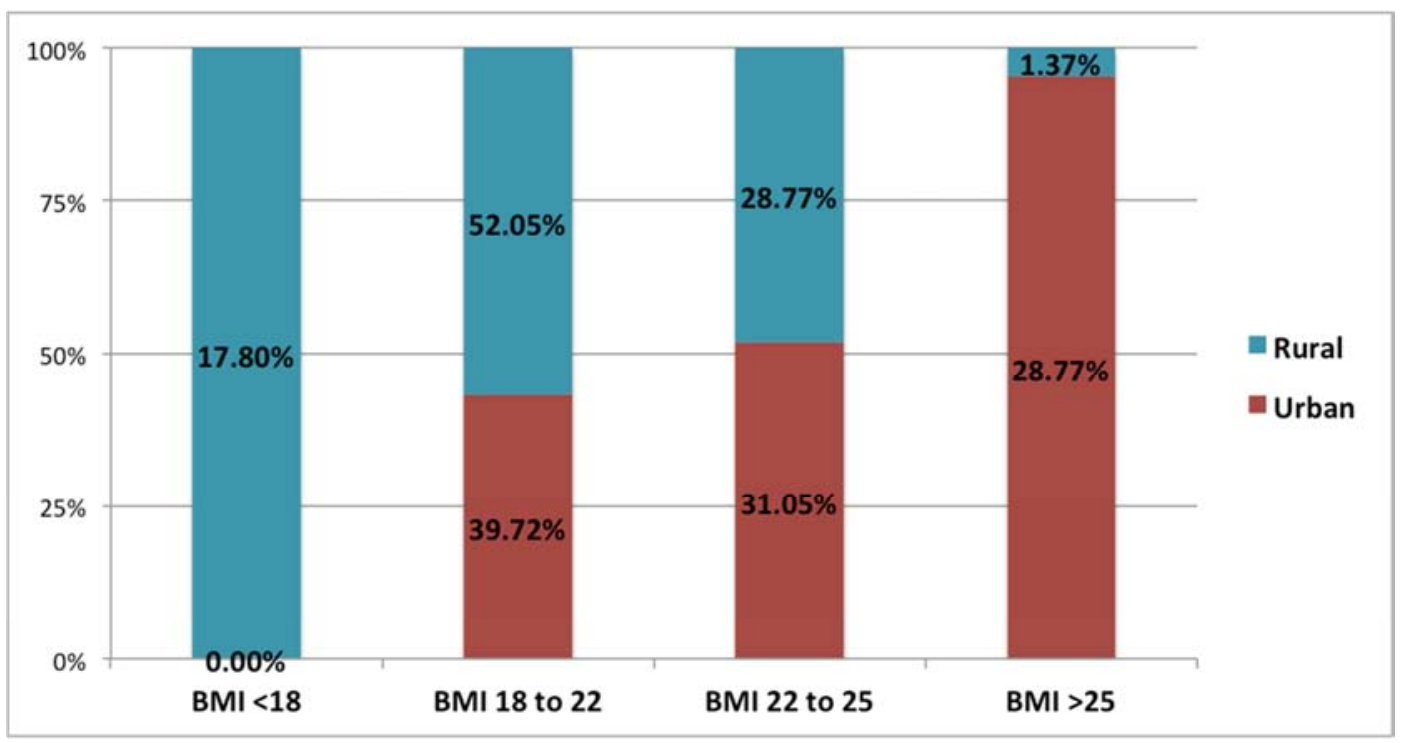

Figure 2. BMI values and percentage distribution of participants.

Mean body fat percentage $(\mathrm{BF} \%)$ is significantly higher in all subgroups from urban areas $(\mathrm{P}<0.001)$ comparing to those from rural settings. Mean energy intake (EI) is found to be significant only in the subgroup of 31-45 years between urban and rural areas. Mean BMR is significantly $(\mathrm{P}<0.001)$ lower in all subgroups from rural settings. No significance was observed in EI:BMR ratios. Mean EI:BMR ratio was over 1.4 in all subgroups from both urban and rural settings except for 46-60 years participants from urban areas. The highest EI:BMR ratio has been observed in the subgroup of 31-45 years aged women from urban areas (table 4).

Table 5. Comparison of body composition values.

\begin{tabular}{lllllll}
\hline & Urban 15-30 (yrs) & Rural 15-30 (yrs) & Urban 31-45 (yrs) & Rural 31-45 (yrs) & Urban 46-60 (yrs) & Rural 46-60 (yrs) \\
\hline BF\% & 22.36 & 11.73 & 21.2 & 12.64 & 14.69 & 11.17 \\
EI & 1744 & 1680 & 2030 & 1535 & 1280 & 1259 \\
BMR & 1186.62 & 1023.62 & 1212.87 & 977.5 & 1072.25 & 817.87 \\
EI:BMR & 1.47 & 1.64 & 1.67 & 1.57 & 1.19 & 1.54 \\
\hline
\end{tabular}

\section{Discussion}

Socio-economic status was addressed depending on family income level. In Bangladesh though the situation has changed in past two decades yet a major portion of the women living in rural areas depend on the male member of the society for financial support. This situation differs in urban areas, as a major portion of the women living in urban areas is either government or non-government jobholders
[15]. But for women with mental illness, they have to depend solely on the family, which is true for participants from both urban and rural settings. Age of onset of mental impairment is found to be significantly higher from 31-45 years in both urban and rural settings. A major portion from both settings also falls into the 15-30 years age limit. The lowest number falls in 46-60 years age limit. This may indicate an early onset of mental impairment among women despite of their socio-demographic status.

In rural settings, over half of the total study participants 
are either currently married or have been married at some point of their life. This number is significantly low among participants from urban settings comparing that of rural areas Divorce rates are much higher in both urban and rural areas for women with mental impairment who has got married, $36.84 \%$ and $43.59 \%$ respectively. Among the women who have been married $84.61 \%$ from rural areas and $63.15 \%$ from urban areas have one or more children. Pregnancy rates among women with mental impairment seem to be much higher among participants living in rural areas.

For study participants both from urban and rural settings prevalence of multiple chronic conditions is very high. This study has identified that though mentally impaired women from rural settings have higher prevalence of chronic disease they are less taken care of. Often this is due to negligence from family members or sometimes due to financial inability of the family. Therefore, a major portion of mentally impaired women from rural settings has undiagnosed underlying chronic diseases. Prevalence of skin diseases is found to be very common among study participants from both settings. An explanation to this could be their lack of self-hygiene and cleanliness. This study has identified that many of these women are not under regular medical follow up or medication despite of their ongoing physical symptoms

The study has identified differences in dietary intake depending on socio-demographic status. Mentally impaired women from rural settings have low daily food consumption and this group lacks in dietary patterns comparing to urban settings in average protein, fat and vitamin consumption. Only in case of carbohydrate consumption not much discrepancy is observed in this study among urban and rural women with mental impairment. Energy intake is considerably higher among women with mental impairment from urban areas comparing to those from rural settings. This finding is somewhat similar as reported in other studies conducted among healthy women from these two settings [15 20].

The study has identified significant differences in BMI among participants from urban and participants from rural settings. Mentally impaired women from urban settings have a higher BMI than those of rural areas. Higher percentage of low BMI among mentally impaired women from rural settings indicates inferior nutritional status of this group. Interestingly over weight among mentally impaired women from urban settings has been observed in this study. A significant portion of mentally impaired women (28.77\%) from urban settings is found to have over weight. Mean body fat percentage is significantly higher among mentally impaired women from urban settings than those from rural areas. But when BMI and BF\% from both settings in this study has been compared to those of healthy women found in previous studies, both $\mathrm{BMI}$ and $\mathrm{BF} \%$ is significantly low among this study participants [15]. Lower BMI and BF\% among mentally impaired women compared to those of healthy women from urban and rural settings highlights inferior nutritional status among this group despite of their socio-demographic status.
Minimum energy expenditure of an individual when not engaged in any regular physical labour is 1.4 times the BMR [21]. Except for one subgroup in this study all other subgroups reflect this hypothesis. For the women from 46-60 years in urban settings having a EI:BMR ratio of 1.19 might be due to reduction of energy intake on the day of data collection.

\section{Conclusion}

Several studies have indicated prevalence of lower nutritional status among healthy women from lower income groups or from rural areas of Bangladesh. Though study participants in this study were mentally impaired yet the findings are similar to those of healthy women in urban and rural settings. Alarmingly mentally impaired women even from well off families or from urban settings have an inferior nutritional status comparing to those of healthy women from urban settings or below standard nutritional status of the women in this region. The nutritional status of mentally impaired women in rural areas or from lower sociodemographic settings is even worse. Low BMI and low energy intake indicates vulnerability of mentally impaired women to malnutrition in Bangladesh. The findings of this study ask for major reform in health system to provide better support and care for mentally impaired population and sustainable initiatives to uplift nutritional status of mentally impaired women at risk in Bangladesh and other developing countries.

\section{References}

[1] Global Alliance for Vaccines and Immunisation, GAVI Country Factsheet: Bangladesh. 2016, Global Alliance for Vaccines and Immunisation.

[2] World Health Organisation, EPI Fact Sheet: Bangladesh 2014. 2014, World Health Organisation.

[3] Food and Agriculture Organisation of the United Nations (FAO), Nutrition Country Profiles, Bangladesh. 2010.

[4] Rashid, M. and S. J. Ulijaszek, Daily energy expenditure across the course of lactation among urban Bangladeshi women. American journal of physical anthropology, 1999. 110 (4): p. 457-465.

[5] Vinoy, S., L. Rosetta, and C. Mascie-Taylor, Repeated measurements of energy intake, energy expenditure and energy balance in lactating Bangladeshi mothers. European journal of clinical nutrition, 2000. 54 (7): p. 579-585.

[6] Prince, M., et al., No health without mental health. The lancet, 2007. 370 (9590): p. 859-877.

[7] Koo, J.-R., et al., Association of depression with malnutrition in chronic hemodialysis patients. American journal of kidney diseases, 2003. 41 (5): p. 1037-1042.

[8] Walters, B. A., et al., Health-related quality of life, depressive symptoms, anemia, and malnutrition at hemodialysis initiation. American Journal of Kidney Diseases, 2002. 40 (6): p. 1185-1194. 
[9] Johansson, Y., et al., Malnutrition in a home - living older population: prevalence, incidence and risk factors. A prospective study. Journal of clinical nursing, 2009. 18 (9): p. $1354-1364$.

[10] Smoliner, C., et al., Malnutrition and depression in the institutionalised elderly. British Journal of Nutrition, 2009. 102 (11): p. 1663-1667.

[11] Hossain, M. D., et al., Mental disorders in Bangladesh: a systematic review. BMC psychiatry, 2014. 14 (1): p. 1.

[12] World Health Organisation, Mental Health System in Bangladesh. 2007, World Health Organisation: Bangladesh.

[13] Chowdhury, A., M. Salim, and N. Sakeb, Some aspects of psychiatric morbidity in the out-patient population of a general hospital. Bangladesh Medical Research Council Bulletin, 1975. 1 (1): p. 51-59.

[14] Hosain, G. M., et al., Prevalence, pattern and determinants of mental disorders in rural Bangladesh. Public Health, 2007. 121 (1): p. 18-24.

[15] Islam, M. Z., M. Akhtaruzzaman, and C. Lamberg-Allardt, Nutritional status of women in Bangladesh: comparison of energy intake and nutritional status of a low income rural group with a high income urban group. Asia Pacific journal of clinical nutrition, 2004. 13 (1): p. 61-68.

[16] Hossain, B., et al., Nutritional Status of Pregnant women in selected rural and urban area of Bangladesh. Journal of Nutrition \& Food Sciences, 2013. 2013.

[17] World Health Organisation, Physical status: the use and interpretation of anthropometry. 1995.

[18] Mifflin, M. D., et al., A new predictive equation for resting energy expenditure in healthy individuals. The American journal of clinical nutrition, 1990. 51 (2): p. 241-247.

[19] Deurenberg, P., J. A. Weststrate, and J. C. Seidell, Body mass index as a measure of body fatness: age-and sex-specific prediction formulas. British journal of nutrition, 1991. 65 (02): p. 105-114.

[20] Jahan, K., Nutrition Survey of Bangladesh 1995-96. Dhaka: Institute of Nutrition and Food Science, Dhaka University, 1996.

[21] WHO/FAO/UNU Expert Consultation, Human energy requirements. 2001: Rome. 